\title{
Effectiveness of strengthened stimulation during acupuncture for the treatment of Bell palsy: a randomized controlled trial
}

\author{
Sha-bei Xu MD PhD, Bo Huang MD PhD, Chen-yan Zhang MMed, Peng Du MD PhD, Qi Yuan MMed, \\ Gui-juan Bi MMed, Gui-bin Zhang MD PhD, Min-jie Xie PhD, Xiang Luo MD, Guang-ying Huang MD PhD, \\ Wei Wang MD PhD
}

See related editorial by Fletcher on page 459 and at www.cmaj.ca/lookup/doi/10.1503/cmaj.130319

\begin{abstract}
Background: The traditional Chinese theory of acupuncture emphasizes that the intensity of acupuncture must reach a threshold to generate de qi, which is necessary to achieve the best therapeutic effect. De qi is an internal compound sensation of soreness, tingling, fullness, aching, cool, warmth and heaviness, and a radiating sensation at and around the acupoints. However, the notion that de qi must be achieved for maximum benefit has not been confirmed by modern scientific evidence.
\end{abstract}

Methods: We performed a prospective multicentre randomized controlled trial involving patients with Bell palsy. Patients were randomly assigned to the de qi $(n=167)$ or control $(n=171)$ group. Both groups received acupuncture: in the de qi group, the needles were manipulated manually until de qi was reached, whereas in the control group, the needles were inserted without any manipulation. All patients received prednisone as a basic treatment. The primary outcome was facial nerve function at month 6 . We also assessed disability and quality of life 6 months after randomization.

Results: After 6 months, patients in the de qi group had better facial function (adjusted odds ratio [OR] 4.16, 95\% confidence interval [CI] 2.23-7.78), better disability assessment (differences of least squares means $9.80,95 \%$ Cl 6.2913.30) and better quality of life (differences of least squares means $29.86,95 \% \mathrm{Cl} 22.33-37.38$ ). Logistic regression analysis showed a positive effect of the de qi score on facial-nerve function (adjusted OR 1.07, 95\% Cl 1.04-1.09).

Interpretation: Among patients with Bell palsy, acupuncture with strong stimulation that elicited de qi had a greater therapeutic effect, and stronger intensity of de qi was associated with the better therapeutic effects. Trial registration: Clinicaltrials.gov no. NCT00685789.
$\mathrm{T}$ he use of acupuncture has gained increasing attention worldwide..$^{1,2}$ There is a long-held belief in the traditional theory and clinical practice of acupuncture that the intensity of the stimulus must reach a threshold to elicit de qi, which plays a pivotal role in achieving the best therapeutic effects. ${ }^{3,4}$ De $q i-$ an internal compound sensation of soreness, tingling, fullness, aching, cool, warmth, heaviness and a radiating sensation at and around acupoints - is elicited by manipulation of the needles (rotated as well as being moved upward and downward). ${ }^{5}$ The concept of de qi, originating from the long-term accumulation of clinical experiences, has been explained to some degree by several scientific studies. ${ }^{6-11}$ However, this long-held belief has not been confirmed by sufficient evidence from randomized controlled trials. ${ }^{5}$ Consequently, de qi and its related techniques have been neglected in acupuncture practice and research. As such, the therapeutic effects of acupuncture may be seriously compromised. ${ }^{12-14}$

In this study, we compared the efficacy of acupuncture with either strong (intended to elicit de qi) or weak stimulation among patients with Bell palsy.

\section{Methods}

\section{Participants}

This study was carried out at 11 tertiary care hospitals in China. Patients were recruited from October 2008 to April 2010, and follow-up was continued until October 2010. All of the hospitals had registered acupuncturists with more than 10 years of professional experience.

To minimize the psychosocial effect of acupuncture, we included patients with Bell palsy, instead of pain-related diseases, because regeneration of facial nerves does not seem to be
Competing interests: None declared.

This article has been peer reviewed.

Correspondence to:

Wei Wang,

wwang_tjh@yahoo.com.cn

CMAJ 2013. DOI:10.1503

/cmaj.121108 
as susceptible to the psychosocial effect as analgesia is. Eligible patients with Bell palsy were given a copy of the informed consent form and those who signed the form were recruited.

The study was approved by all local institutional ethics review boards and was conducted in accordance with the provisions of the Declaration of Helsinki and Good Clinical Practice guidelines. ${ }^{15}$

We included patients who met the following criteria: unilateral facial-nerve weakness without an identifiable cause within 168 hours after the onset of symptoms $;{ }^{16}$ aged $18-65$ years; and who had not received any treatment before randomization. We excluded participants who were illiterate; had facial paralysis caused by herpes zoster; had recurrent facial paralysis; had noticeable asymmetry of the face before the onset of illness (which may affect evaluation); and who had a history of peptic ulcer disease, severe hypertension, uncontrolled diabetes, liver and kidney dysfunction, mental illness or serious systemic diseases that may affect the treatment. We also excluded pregnant women.

\section{Interventions}

All acupuncturists $(n=15)$ were registered acupuncturists with more than 10 years of professional experience and had been trained in the standardized procedures by an acupuncture expert. During the treatment process, the acupuncturists explained "acupuncture" and "de qi" to patients in both groups in the same way. The acupuncture intervention was based on traditional Chinese acupuncture theory and previous Chinese literature. ${ }^{17}$ Acupoints included Yangbai (gallbladder meridian 14, affected side), Dicang (stomach meridian [ST] 4, affected side), Jiache (ST 6, affected side), Xiaguan (ST 7, affected side), Yifeng (triple energizer meridian 17, affected side) and Hegu (large intestine meridian 4, contralateral). For both groups, treatment consisted of twenty 30-minute sessions (5 sessions per wk for $4 \mathrm{wk})$. The same number and type of needles $(\Phi=0.25 \mathrm{~mm} \times 25 \mathrm{~mm}$ disposable sterile acupuncture needles, Shanghai Taicheng Technology and Development Co., Ltd.) were used for both groups. All patients received prednisone therapy as a basic treatment for 14 days $(30 \mathrm{mg} / \mathrm{d}$ for $7 \mathrm{~d}, 20 \mathrm{mg} / \mathrm{d}$ for $4 \mathrm{~d}$ and $10 \mathrm{mg} / \mathrm{d}$ for $3 \mathrm{~d}$ ).

In the $d e q i$ group, the needles were manipulated manually after insertion, using techniques such as lifting, thrusting and twirling, until de $q i$ was achieved. The needles were left in place for 30 minutes. During the treatment session, the acupuncturist asked the patient about de qi every 10 minutes and manipulated the needles to maintain the intensity of the sensation. In the control group, the needles were inserted and left in place for 30 minutes without manipulation. Patients were kept out of contact with those in the opposite treatment group. All sessions for each patient were performed by the same acupuncturist.

\section{Outcomes}

The primary outcome was facial-nerve function measured by use of the House-Brackmann score. ${ }^{18}$ The House-Brackmann scale is based on a sixgrade score: grade 1 indicates normal function and grade 6 indicates complete paralysis. Patients were asked to show 4 standard facial expressions: at rest, raised eyebrows, eyes tightly closed, and showing teeth, which were recorded by use of a digital camcorder. All digital data were assessed and graded independently by 3 neurologists at Tongji Hospital; the neurologists were unaware of the study-group assignments and the stage of assessment.

The secondary outcomes were disability (measured by the Facial Disability Index) and health-related quality of life (measured by the World Health Organization's Quality of Life, brief version). ${ }^{19,20}$

Baseline assessment was performed on the day of randomization, and follow-up visits were conducted 6 months after randomization.

\section{Assessment of de qi}

Immediately after removing the needles during the 1st, 5th, 10th, 15th and 20th sessions, patients were interviewed by another researcher on the team who did not know the treatment allocation. Patients were questioned about whether each of the 8 sensa-

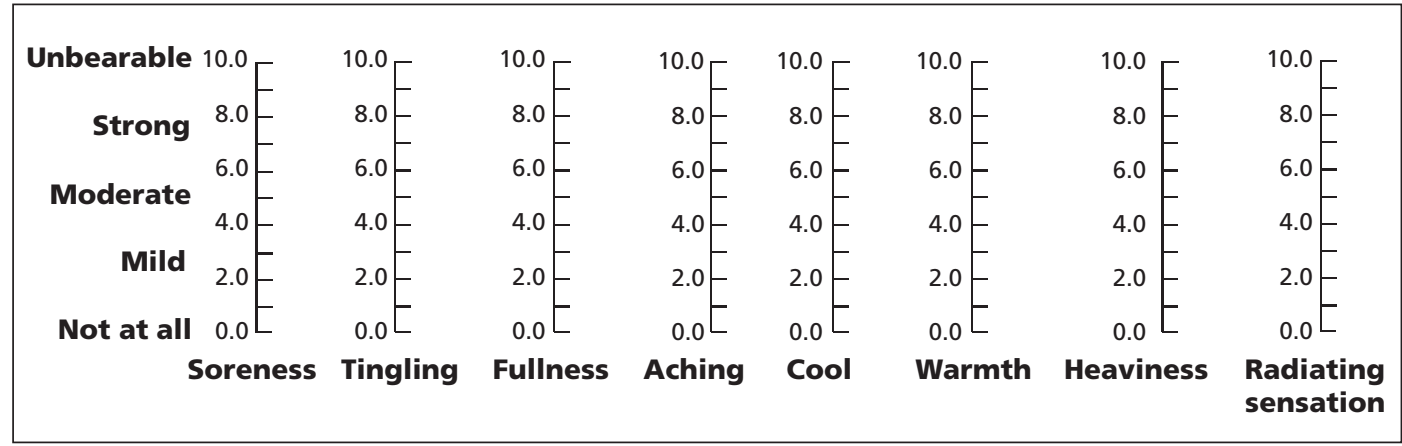

Figure 1: The visual analogue scale used to assess the intensity of de qi. 
tions of de qi had occurred during the session. Intensity was rated using a visual analogue scale (Figure 1), which has commonly been used to measure the intensity of $d e q i^{21-23}$ The patient was asked to indicate his or her response to each sensation anywhere on the continuum. The score for the visual analogue scale was the sum of all 8 component scores. The overall de qi score was the mean score from all sessions.

\section{Sample size}

Before the study, we assumed that $80 \%$ of patients would recover completely in the control group,${ }^{24}$ compared with $90 \%$ of patients in the de $q i$ group. To detect a statistically significant difference between the 2 groups with at least $80 \%$ power at a $5 \%$ significance level, we planned to include 487 patients, which included a possible loss to follow-up of $10 \%$.

\section{Randomization, implementation and blinding}

To avoid bias, we stratified patients according to the interval between the onset of palsy and the start of treatment $(\leq 72 \mathrm{~h}$ or $72-168 \mathrm{~h}$ ), score on the House-Brackmann Scale (2-3 or 4-5) and treatment centre. After a senior neurologist who had been trained in the study procedures confirmed eligibility and written informed consent was obtained, patients were randomly assigned to either the de qi or control group via computergenerated random numbers. The treatment allocations were sealed in opaque envelopes that were opened sequentially. A third party, who was not involved in the trial, produced the randomization codes and the sealed envelopes. One person at each hospital was responsible for the envelopes. The senior neurologist, who confirmed eligibility of the patient, determined which stratum (HouseBrackmann score and interval between onset of palsy and start of treatment) the patient belonged to. Then, the person in charge of the envelopes would give the specified envelope to the acupuncturist. To preserve masking, only the acupuncturists had access to the treatment allocation. The patients, recruiters and outcome assessors were all unaware of study-group assignments.

\section{Statistical analysis}

We used two-tailed tests for all statistical analyses. Statistical significance was defined as $p<0.05$. Quantitative data are presented as mean ( \pm standard deviation [SD]), and qualitative data by frequency and percentage. We compared the rates of

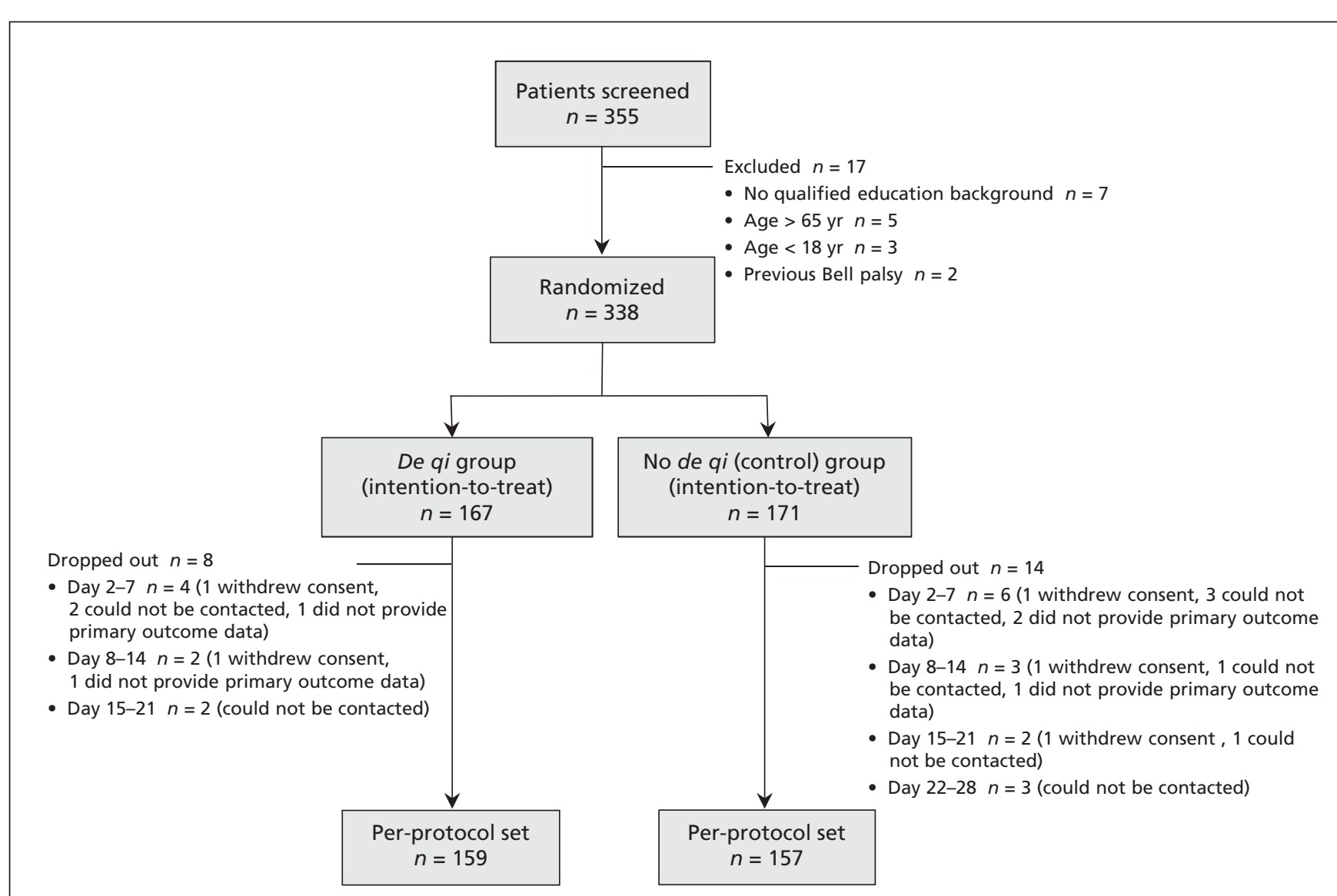

Figure 2: Flowchart of trial participants. 
facial-nerve function (grade 1 on the HouseBrackmann Scale) at 6 months between the control and treatment groups by logistic regression analysis; age, sex, treatment centre, interval between the onset of palsy and the start of treatment, and House-Brackmann score on day 1 were included

Table 1: Baseline characteristics of the included patients

\begin{tabular}{|c|c|c|}
\hline \multirow[b]{2}{*}{ Characteristic } & \multicolumn{2}{|c|}{ No. $(\%)$ of patients* } \\
\hline & $\begin{array}{c}\text { De qi group } \\
\quad n=167\end{array}$ & $\begin{array}{c}\text { Control group } \\
\quad n=171\end{array}$ \\
\hline Women & $69(41.3)$ & $76(44.4)$ \\
\hline Men & $98(58.7)$ & $95(55.6)$ \\
\hline Age, yr, mean \pm SD & $38.12 \pm 12.76$ & $38.15 \pm 12.75$ \\
\hline \multicolumn{3}{|c|}{ Interval between onset of palsy and start of treatment } \\
\hline$\leq 72 \mathrm{~h}$ & $108(64.7)$ & $98(57.3)$ \\
\hline$>72$ to $\leq 168 \mathrm{~h}$ & $59(35.3)$ & $73(42.7)$ \\
\hline \multicolumn{3}{|l|}{ House-Brackmann score† } \\
\hline 2 & $8(4.8)$ & $10(5.9)$ \\
\hline 3 & $88(52.7)$ & $93(54.4)$ \\
\hline 4 & $54(32.3)$ & $57(33.3)$ \\
\hline 5 & $17(10.2)$ & $11(6.4)$ \\
\hline \multicolumn{3}{|c|}{ Facial Disability Index score, mean \pm SD } \\
\hline Physical function subscale & $64.82 \pm 14.13$ & $67.84 \pm 12.88$ \\
\hline Social/well-being subscale & $65.58 \pm 14.82$ & $67.58 \pm 12.46$ \\
\hline \multicolumn{3}{|c|}{ WHO Quality of Life (brief version) score, mean \pm SD } \\
\hline Physical domain & $63.17 \pm 13.16$ & $63.30 \pm 12.29$ \\
\hline Psychological domain & $59.47 \pm 10.98$ & $60.24 \pm 10.36$ \\
\hline Social domain & $65.87 \pm 14.00$ & $67.93 \pm 11.82$ \\
\hline Environment domain & $60.78 \pm 12.21$ & $60.71 \pm 12.16$ \\
\hline \multicolumn{3}{|l|}{ Education } \\
\hline Junior middle school & $47(28.1)$ & $49(28.7)$ \\
\hline Senior middle school & $41(24.6)$ & $41(24.0)$ \\
\hline Junior college & $17(10.2)$ & $19(11.1)$ \\
\hline Bachelor & $59(35.3)$ & $58(33.9)$ \\
\hline Postgraduate & $3(1.8)$ & $4 \quad(2.3)$ \\
\hline \multicolumn{3}{|l|}{ Occupation } \\
\hline Civil servant & $5(3.0)$ & $6(3.5)$ \\
\hline Technician & $33(19.8)$ & $35(20.5)$ \\
\hline Clerk & $27(16.2)$ & $30(17.5)$ \\
\hline Service & $36(21.6)$ & $24(14.0)$ \\
\hline Worker & $10(6.0)$ & $13(7.6)$ \\
\hline Student & $32(19.2)$ & $33(19.3)$ \\
\hline Unemployed or retiree & $24(14.4)$ & $30(17.5)$ \\
\hline \multicolumn{3}{|l|}{ Affected side } \\
\hline Left & $85(50.9)$ & $84(49.1)$ \\
\hline Right & $82(49.1)$ & $87(50.9)$ \\
\hline
\end{tabular}

as covariables. We also calculated odds ratios (ORs) and 95\% confidence intervals (CIs). Scores on the Facial Disability Index and the Quality of Life scale (brief version) at 6 months were secondary outcomes and were compared between groups by analysis of covariance (covariables: age, sex, treatment centre, interval between the onset of palsy and the start of treatment, House-Brackmann score on day 1, and Facial Disability Index score or Quality of Life score (brief version) on day 1 , respectively). Differences of least squares means and $95 \%$ CIs were calculated.

Interaction terms for group and centre were added in the logistic regression model for the primary outcome and in the analysis of covariance models for the secondary outcomes. Logistic regression analysis was used to study the influence of the sum scores of de qi as continuous variable on the rates of grade 1 scores on the House-Brackmann Scale at 6 months; the analysis was adjusted for age, sex, treatment centre, interval between the onset of palsy and the start of treatment, and House-Brackmann score on day 1 . We use the last observation carried forward to impute missing data.

\section{Results}

Of the 355 patients referred for participation in the study, $338(95.2 \%)$ were assigned to a treatment group. Final outcomes were available for 316 patients $(93.5 \%)$ (Figure 2). Patient recruitment was stopped before the intended sample size was reached because the research grant expired. The decision to stop recruitment was made by the trial steering committee on administrative grounds without knowledge of the data.

The baseline characteristics of the de $q i$ and control groups were similar $(p>0.05$; Table 1$)$. The following tests for consistency found no significant difference between centres: grade 1 scores on the House-Brackmann scale (intention-to-treat: $p=0.5$; per protocol: $p=0.4$ ); scores on the Facial Disability Index (intention-to-treat: $p=0.6$; per protocol: $p=0.4$ ); Quality of Life scale (intentionto-treat: $p=0.2$; per protocol: $p=0.6$ ).

\section{Outcomes}

Our analyses were based on the intention-to-treat principle using the imputed data set $(n=338)$ and the per-protocol data set $(n=316)$. At 6 months, facial-nerve function, disability and quality of life were significantly better in the de $q i$ group than in the control group (Table 2).

\section{Additional analyses}

Of the 316 patients who completed the 6-month follow-up visit, $262(82.9 \%)$ rated de $q i$ on the visual analogue scale, and the other 54 (17.1\%) 
stated that they could not understand or differentiate between the 8 elements of $d e$ qi and thus did not rate it. Of these patients, 24 were in the de qi group and 30 were in the control group.

The overall de qi score was significantly higher in the de $q i$ group ( $n=135$; mean $22.74[ \pm 3.56 \mathrm{SD})$ than in the control group $(n=127 ; 14.85[ \pm 2.61])(p<0.001)$. When we set the threshold for de qi at a score of 20 on the visual analogue scale (using the assessment method proposed by Hui and colleagues $\left.{ }^{21}\right)$, de qi was achieved for $80.0 \%(108 / 135)$ of patients in the de qi group and $1.6 \%(2 / 127)$ of patients in the control group $(p<0.001)$.

Among the 262 patients who rated de qi on the visual analogue scale, there was a positive effect of de qi on grade 1 scores on the HouseBrackmann scale at 6 months (adjusted OR 1.07, 95\% CI 1.04-1.09).

\section{Safety}

No serious adverse events were reported. In total, 212 adverse events were reported in the de qi group ( 94 cases of minor bleeding, 73 cases of needling pain, 43 cases of bruising and 2 cases of faintness during treatment), and 203 adverse events were reported in the control group (83 cases of minor bleeding, 78 cases of needling pain, 38 cases of bruising, 3 cases of faintness during treatment and 1 case of drowsiness). All participants recovered fully from the adverse events.

\section{Interpretation}

We found evidence that acupuncture with de qi improved facial muscle recovery, disability and quality of life among patients with Bell palsy. Stronger intensity of de qi was associated with better therapeutic effects. Examination of videotaped

\begin{tabular}{|c|c|c|c|}
\hline \multirow[b]{2}{*}{ Variable } & \multicolumn{2}{|c|}{ Mean $(95 \% \mathrm{Cl})$ * } & \multirow{2}{*}{$\begin{array}{c}\text { Adjusted } t \\
\text { differences of least } \\
\text { squares means } \\
(95 \% \mathrm{Cl})^{*}\end{array}$} \\
\hline & $\begin{array}{l}\text { De qi group } \\
\quad n=167\end{array}$ & $\begin{array}{l}\text { Control group } \\
\quad n=171\end{array}$ & \\
\hline \multicolumn{4}{|l|}{ Intention-to-treat analysis } \\
\hline Complete recovery, no. (\%)‡ & $150(89.8)$ & $121(70.8)$ & $4.2(2.2-7.8) \S$ \\
\hline Facial Disability Index & $191.8(189.3-194.3)$ & $182.0(179.6-184.5)$ & $9.8(6.3-13.3)$ \\
\hline Physical function & $98.2 \quad(96.8-99.5)$ & $94.8 \quad(93.5-96.1)$ & $3.4(1.5-5.3)$ \\
\hline Social function & $93.6 \quad(92.2-95.1)$ & $87.2 \quad(85.8-88.7)$ & $6.4 \quad(4.3-8.4)$ \\
\hline $\begin{array}{l}\text { WHO Quality of Life (brief } \\
\text { version) score }\end{array}$ & $340.1(334.8-345.4)$ & $310.3(305.0-315.5)$ & $29.9(22.3-37.4)$ \\
\hline Physical domain & $93.8 \quad(92.1-95.4)$ & $86.2 \quad(84.6-87.8)$ & $7.6 \quad(5.3-9.9)$ \\
\hline Psychological domain & $80.5 \quad(79.3-81.8)$ & $73.0 \quad(71.8-74.2)$ & $7.5 \quad(5.8-9.3)$ \\
\hline Social domain & $85.6 \quad(83.9-87.2)$ & $85.6 \quad(83.9-87.2)$ & $7.2(4.8-9.5)$ \\
\hline Environment domain & $80.2 \quad(78.7-81.7)$ & $72.7 \quad(71.2-74.2)$ & $7.5 \quad(5.3-9.6)$ \\
\hline \multicolumn{4}{|l|}{ Per-protocol analysis } \\
\hline Complete recovery, no. (\%)‡ & $150(94.3)$ & $121(77.1)$ & $6.0 \quad(2.7-13.6) \S$ \\
\hline Facial Disability Index & $194.8(193.4-196.1)$ & $185.8(184.5-187.2)$ & $9.0(7.0-10.9)$ \\
\hline Physical function & $99.8 \quad(99.3-100.3)$ & $97.0 \quad(96.5-97.5)$ & $2.8(2.0-3.5)$ \\
\hline Social function & $95.0 \quad(93.8-96.1)$ & $88.9 \quad(87.7-90.0)$ & $6.1 \quad(4.4-7.8)$ \\
\hline $\begin{array}{l}\text { WHO Quality of Life (brief } \\
\text { version) score }\end{array}$ & $345.1(340.5-349.7)$ & $315.0(310.4-319.6)$ & $30.2(23.6-36.7)$ \\
\hline Physical domain & $95.5 \quad(94.1-96.8)$ & $88.0 \quad(86.7-89.4)$ & $7.4 \quad(5.6-9.3)$ \\
\hline Psychological domain & $81.7 \quad(80.6-82.8)$ & $74.0 \quad(72.9-75.1)$ & $7.7 \quad(6.2-9.3)$ \\
\hline Social domain & $86.7 \quad(85.2-88.2)$ & $79.4 \quad(77.9-81.0)$ & $7.3 \quad(5.1-9.5)$ \\
\hline Environment domain & $81.2 \quad(79.8-82.7)$ & $73.7 \quad(72.2-75.1)$ & $7.6 \quad(5.5-9.6)$ \\
\hline \multicolumn{4}{|c|}{$\begin{array}{l}\text { Note: } \mathrm{Cl}=\text { confidence interval, } \mathrm{OR}=\text { odds ratio, } \mathrm{WHO}=\text { World Health Organization. } \\
* \text { *Unless stated otherwise. } \\
\text { †Adjusted for age, sex, treatment centre, interval between onset of palsy and start of treatment, House-Brackmann score on } \\
\text { day 1, and baseline score on the corresponding parameters (the scores on Facial Disability Index or its subscales, or the scores on } \\
\text { World Health Organization Quality of Life, brief version or its subscales). } \\
\text { ‡Grade } 1 \text { on the House-Brackmann scale. } \\
\text { \$Adjusted odds ratios (95\% confidence intervals); adjusted for age, sex, treatment centre, interval between onset of palsy and } \\
\text { start of treatment, and House-Brackmann score on day } 1 .\end{array}$} \\
\hline
\end{tabular}


facial movements by an expert panel confirmed that the findings were consistent between the 11 included hospitals.

There has been controversy about whether much of the clinical benefit of acupuncture is because of the powerful psychologic effect. A systematic review of clinical trials reported that placebos had no significant effect on objective continuous outcomes, ${ }^{25}$ and a recent systematic review of pooled data from meta-analyses suggests that acupuncture has a greater effect than placebo. ${ }^{26} \mathrm{In}$ our study, we used objective functional outcomes instead of a subjective outcome such as pain. Thus, the placebo effect was minimized.

In the promotion and spread of acupuncture, the individualized, humanized and interactive process of traditional acupuncture has often been misconstrued as a fixed, mechanized and oneway approach. Therefore, the best therapeutic effects of acupuncture often are not achieved in clinical practice. The negative findings of some randomized controlled trials on acupuncture might be because the procedural techniques were inadequate and the intensity of needle sensation was not sufficient. Of the 49 guidelines for acupuncture treatment in labour and postnatal wards in Sweden, only 2 guidelines mentioned the importance of achieving de qi. ${ }^{12} \mathrm{~A}$ narrative review reported that only 6 out of the 47 included systematic reviews applied some criteria for the adequacy of acupuncture treatment. ${ }^{14}$

\section{Limitations}

Our study had several limitations. We did not use sham acupuncture in the control (weak-stimulation) group because Chinese people generally trust in acupuncture, and informed consent for the use of sham acupuncture is very difficult to obtain from these patients. However, several clinical trials have shown that real acupuncture needling, especially with rotation, can elicit higher intensity sensations than sham or superficial needling. $5,7,21,23,27$ Our observed rate of complete recovery in the control group ( $77.1 \%$ of patients) is similar to that in the corticosteroid group $(76.7 \%)$ in a recent Cochrane review, ${ }^{28}$ which suggests that patients in our control group had similar experiences to patients who received sham acupuncture.

Another limitation was that $17.1 \%$ of patients did not rate de $q i$, although we excluded patients who were illiterate. Thus, the findings about the association between the intensity of de qi and therapeutic effect must be interpreted with caution.

\section{Conclusion}

For patients with Bell palsy treated with prednisone, the addition of strong-stimulation acupuncture, which can elicit a higher intensity of de qi, significantly improved the therapeutic effect. $D e q i$ and its related techniques should be properly appreciated in acupuncture practice and research, and should be considered for inclusion in clinical guidelines for acupuncture.

\section{References}

1. Sung JJ. Acupuncture for gastrointestinal disorders: myth or magic. Gut 2002;51:617-9.

2. Han JS. Acupuncture and endorphins. Neurosci Lett 2004;361: 258-61.

3. Johnson MI, Benham AE. Acupuncture needle sensation: the emerging evidence. Acupunct Med 2010;28:111-4.

4. Kong J, Gollub R, Huang T, et al. Acupuncture de qi, from qualitative history to quantitative measurement. J Altern Complement Med 2007; 13:1059-70.

5. Benham A, Phillips G, Johnson MI. An experimental study on the self-report of acupuncture needle sensation during deep needling with bi-directional rotation. Acupunct Med 2010;28:16-20.

6. Hsieh JC, Tu CH, Chen FP, et al. Activation of the hypothalamus characterizes the acupuncture stimulation at the analgesic point in human: a positron emission tomography study. Neurosci Lett 2001; 307:105-8.

7. Wu MT, Hsieh JC, Xiong J, et al. Central nervous pathway for acupuncture stimulation: localization of processing with functional MR imaging of the brain — preliminary experience. Radiology 1999;212:133-41.

8. Takamoto K, Hori E, Urakawa S, et al. Cerebral hemodynamic responses induced by specific acupuncture sensations during needling at trigger points: a near-infrared spectroscopic study. Brain Topogr 2010;23:279-91.

9. Yin CS, Park HJ, Kim SY, et al. Electroencephalogram changes according to the subjective acupuncture sensation. Neurol Res 2010;32(Suppl 1):31-6.

10. Sandberg M, Lundeberg T, Lindberg LG, et al. Effects of acupuncture on skin and muscle blood flow in healthy subjects. Eur J Appl Physiol 2003;90:114-9.

11. Lee S, Chae Y, Kim SN, et al. Short term effects by acupuncture to SP3 on the autonomic blood flow control. Neurol Res 2010;32 (Suppl 1):37-42.

12. Schytt E, Halvarsson A, Pedersen-Draper C, et al. Incompleteness of Swedish local clinical guidelines for acupuncture treatment during childbirth. Acta Obstet Gynecol Scand 2011;90: 77-82.

13. Benham A, Johnson MI. Could acupuncture needle sensation be a predictor of analgesic response? Acupunct Med 2009;27:65-7.

14. White A, Cummings M, Barlas P, et al. Defining an adequate dose of acupuncture using a neurophysiological approach - a narrative review of the literature. Acupunct Med 2008;26:111-20.

15. Ministry of Health of the People's Republic of China. Good Clinical Practice [Chinese]. Beijing; The Ministry; 2003. Available: www.moh.gov.cn/mohzcfgs/pgz/201105/51765.shtml (accessed 2013 Feb 8).

16. Gilden DH. Clinical practice. Bell Palsy. N Engl J Med 2004;351: 1323-31.

17. Liang F, Li Y, Yu S, et al. A multicentral randomized control study on clinical acupuncture treatment of Bell palsy. $J$ Tradit Chin Med 2006;26:3-7.

18. House JW, Brackmann DE. Facial nerve grading system. Otolaryngol Head Neck Surg 1985;93:146-7.

19. VanSwearingen JM, Brach JS. The Facial Disability Index: reliability and validity of a disability assessment instrument for disorders of the facial neuromuscular system. Phys Ther 1996;76:128898, discussion 1298-300.

20. World Health Organization. The World Health Organization Quality of Life (WHOQOL) - BREF, Chinese edition. Geneva (Switzerland): The Organization; 2004.

21. Hui KK, Nixon EE, Vangel MG, et al. Characterization of the "Deqi" response in acupuncture. BMC Complement Altern Med 2007;7:33.

22. Kong J, Fufa DT, Gerber AJ, et al. Psychophysical outcomes from a randomized pilot study of manual, electro, and sham acupuncture treatment on experimentally induced thermal pain. J Pain 2005;6:55-64

23. Kou W, Gareus I, Bell JD, et al. Quantification of DeQi sensation by visual analog scales in healthy humans after immunostimulating acupuncture treatment. Am J Chin Med 2007;35:753-65.

24. Salinas RA, Alvarez G, Ferreira J. Corticosteroids for Bell palsy (idiopathic facial paralysis). Cochrane Database Syst Rev 2004;CD001942.

25. Hróbjartsson A, Gøtzsche PC. Is the placebo powerless? An 
analysis of clinical trials comparing placebo with no treatment. N Engl J Med 2001;344:1594-602.

26. Hopton A, MacPherson H. Acupuncture for chronic pain: Is acupuncture more than an effective placebo? A systematic review of pooled data from meta-analyses. Pain Pract 2010;10:94-102.

27. Streitberger K, Kleinhenz J. Introducing a placebo needle into acupuncture research. Lancet 1998;352:364-5.

28. Salinas RA, Alvarez G, Daly F, et al. Corticosteroids for Bell palsy (idiopathic facial paralysis). Cochrane Database Syst Rev 2010;CD001942.

Affiliations: From the Department of Neurology (Xu, B. Huang, C. Zhang, Xie, Luo, Wang), Key Laboratory of Neurological Diseases of Chinese Ministry of Education; Integrated Traditional and Western Medicine (Yuan, Bi, G. Huang), Tongji Hospital, Tongji Medical College, Huazhong University of Science and Technology, Wuhan, Hubei, China; the Department of Neurology (Du), Zhongshan Hospital, Fudan University, Shanghai, China; and the Department of Neurology (G. Zhang), Xiangyang Hospital Affiliated to Hubei University of Medicine, Xiangyang, Hubei, China.

Contributors: Sha-bei $\mathrm{Xu}$ and Bo Huang contributed equally to this article. Wei Wang, Sha-bei Xu, Bo Huang, Chen-yan Zhang, Peng Du, Qi Yuan, Gui-juan Bi, Gui-bin Zhang, Min-jie Xie, Xiang Luo and Guang-ying Huang conceived and designed the study. Wei Wang, Sha-bei Xu and Bo Huang supervised the trial. Sha-bei Xu planned the statistical analyses and wrote the first draft of the manuscript. Bo Huang and Chen-yan Zhang coordinated the trial and collected and analyzed the data. Bo Huang, Chen-yan Zhang,
Peng Du, Qi Yuan, Gui-juan Bi and Gui-bin Zhang recruited patients and ran the trial. Min-jie Xie, Xiang Luo and Guangying Huang contributed to the analysis, discussions and interpretation of data. All of the authors revised the article critically for important intellectual content and approved the final version submitted for publication.

Funding: This work was supported by grants from National Science Fund for Distinguished Young Scholars (no. 30725019), Natural Science Foundation of China (no. 81030021 ) and National Basic Research Program (nos. 2006CB504502, 2011CB504403 and 2011CB505200). The sponsors had no role in the design of the study, the collection, analysis or interpretation of the data, the writing of the manuscript or the decision to submit the article for publication.

Acknowledgements: The authors thank Dr. Zhaori Ge-tu for helpful discussion and Professor Ping Yin for providing statistical support. The authors thank the following investigators who participated in the study: Ling Liu, Hubei Hospital of Traditional Chinese Medicine; Guo-hua Chen, Wuhan No.1 Hospital; Gui-bin Zhang, Xiangyang No.1 People's Hospital; Zhao-hui Zhang, Renmin Hospital of Wuhan University; Xin Wang, Zhongshan Hospital, Fudan University; Jian-ping Wang, The Second Affiliated Hospital, Zhengzhou University; Wei Zhang, Yichang Hospital of Traditional Chinese Medicine; Yuan-wu Mei, Union Hospital, Tongji Medical College, Huazhong University of Science and Technology; Ming-wei Wang, The First Hospital of Hebei Medical University; Hong-hua Li, Wuhan General Hospital of Guangzhou Command PLA.

\section{GET THE CREDIT YOU DESERVE}

New online

accredited CME

courses just released

Free for CMA members

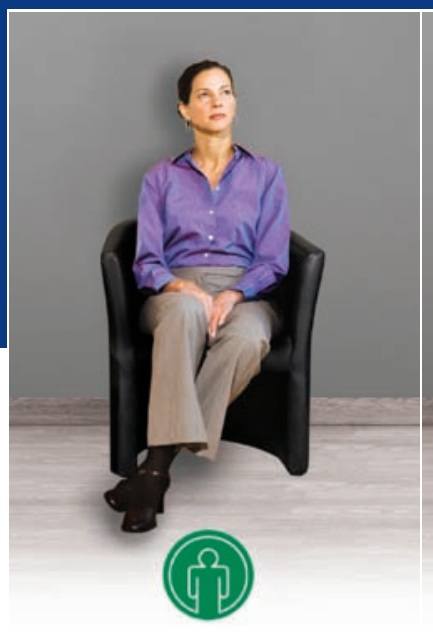

ACUTE/

CHRONIC PAIN

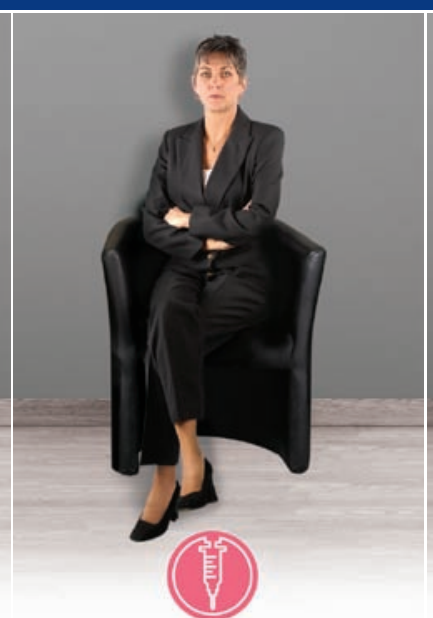

TYPE 2

DIABETES

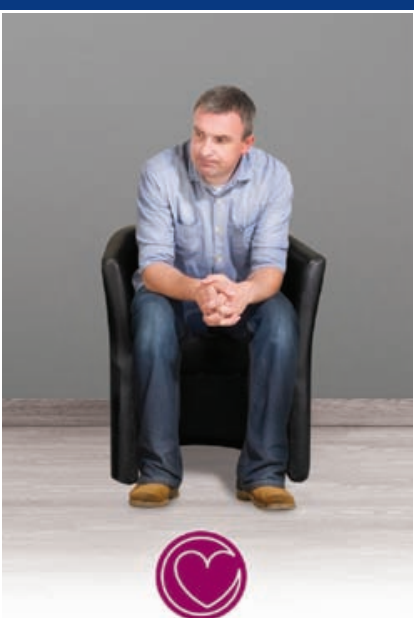

ACUTE CORONARY SYNDROMES
These courses were developed in collaboration with the CMA and Memorial University Professional Development and Conferencing Services through an educational grant from Pfizer Canada.

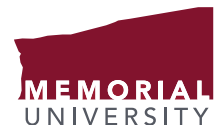

UNIVERSITY 